\title{
SPECIAL RADICALS AND MATRIX NEAR-RINGS
}

\author{
STEFAN VELDSMAN \\ (Received 27 July 1990; revised 18 February 1991) \\ Communicated by P. Schultz
}

\begin{abstract}
Special radical classes of near-rings are defined and investigated. It is shown that our approach, which differs from previous ones, does cater for all the well-known radicals of near-rings. Moreover, most of the desirable properties from their ring theory counterpart are retained. The relationship between the special radical of a near-ring and the corresponding matrix near-ring is given.
\end{abstract}

1991 Mathematics subject classification (Amer. Math. Soc.): 16 Y 30, 16 N 80.

Special radicals in the variety of rings is a specialization of the supernilpotent radicals of rings. The need for supernilpotent radicals arose to discard some pathological radicals. Supernilpotent radicals are hereditary and contain all the nilpotent rings. These two properties (or sometimes just the second) are the defining conditions for a supernilpotent radical in the variety of nearrings. Unfortunately this approach lacks several desirable conclusions, for example, supernilpotent radicals need not have hereditary semisimple classes in the variety of near-rings (cf. [4]). At first it appeared that this is the price to pay for considering supernilpotent radicals in this more general variety. However, recent results seem to indicate that the starting point may not be the correct one. In [17], overnilpotent radical classes of near-rings were defined which also generalize the supernilpotent radicals of rings. The starting point here is via weakly special classes which are classes of quasi semi-equiprime near-rings (defined below). This approach has several advantages; the overnilpotent radicals are always ideal-hereditary. A host of

(C) 1992 Australian Mathematical Society 0263-6115/92 \$A2.00+0.00 
natural examples were given in [17]. In fact, all the known ideal-hereditary radicals are overnilpotent radicals. Whether this is true in general is not yet known.

It is our purpose here to define and investigate special radicals for nearrings as a specialization of overnilpotent radicals. Following the initial definitions and examples, we give in Section 2 a characterization of special radicals via near-ring groups and in the last section we investigate the relationship between the radical of a near-ring and the corresponding matrix near-ring (as defined by Meldrum and van der Walt [9]).

A note of caution: two different meanings have already been assigned to special radicals for near-rings, namely the upper radical determined by a hereditary class which is closed under essential extensions and which consists of prime near-rings [1] or equiprime near-rings [3] respectively. Our definition of a special radical will imply the former and is implied by the latter. The drawbacks of the first definition are the same as those of the supernilpotent radicals of near-rings and the main drawback of the second is that it does not cater for the important $J_{2}$ radical and most probably also not for the Brown-McCoy radical (we do not know if every simple near-ring with identity is equiprime).

All near-rings are $o$-symmetric right near-rings. Most of the present results can be extended to the variety of all, not necessarily $o$-symmetric near-rings along the lines of [17, Section 4]. Pilz [12] can be consulted for undefined near-ring concepts, and [17] or Szasz [13], with their references, for radical theoretic concepts.

\section{Special radicals}

1.1 Definition. A near-ring $N$ is reliable if whenever $\theta: I \rightarrow N$ is a surjective homomorphism and $I \triangleleft A$, then $x-y \in \operatorname{ker} \theta \quad(x, y \in I)$ implies $a x-a y \in \operatorname{ker} \theta$ for all $a \in A$.

If $N$ is reliable, then the $I$-group $I / \operatorname{ker} \theta \cong N$ can be turned into an $A$-group via the natural action: $a n=a\left(i_{n}+\operatorname{ker} \theta\right)=a i_{n}+\operatorname{ker} \theta$. Ideals are denoted by $I \triangleleft N$; essential ideals by $I \triangleleft \circ N \quad(I$ is essential if $I \cap J \neq 0$ for all $0 \neq J \triangleleft N)$. From [17] we recall that a near-rings $N$ is called quasi semi-equiprime if $(0: N)_{N}=0$ and $N$ is reliable. These near-rings $N$ are exactly those with the property that whenever $J \triangleleft I \triangleleft A$ and $I / J \cong N$, then $J \triangleleft A$. A class of near-rings $\mathscr{M}$ is called a weakly special class if it satisfies

(W1) $N \in \mathscr{M}$ implies $N$ is quasi semi-equiprime;

(W2) $\mathscr{M}$ is hereditary (i.e. $I \triangleleft N \in \mathscr{M}$ implies $I \in \mathscr{M}$ ); 
(W3) $\mathscr{M}$ is closed under essential extensions (that is, if $I \triangleleft 0 N$ and $I \in$ $\mathscr{K}$, then $N \in \mathscr{M}$ ).

It is easy to see that $\mathscr{M}$ is weakly special if and only if $\mathscr{M}$ is a hereditary class of quasi semi-equiprime near-rings such that if $I \triangleleft N$ with $I \in \mathscr{M}$ and $(0: I)_{N}=0$, then $I \in \mathscr{M}$. A radical class $\mathscr{R}$ is an overnilpotent radical class if $\mathscr{R}$ is hereditary and $\mathscr{S} \mathscr{R}$, the semisimple class of $\mathscr{R}$, is a hereditary class of qausi semi-equiprime near-rings. The relationship between weakly special classes and overnilpotent radical classes is given by the following: a radical class $\mathscr{R}$ is an overnilpotent radical class if and only if $\mathscr{R}$ is the upper radical class determined by a weakly special $\mathscr{M}$, that is,

$\mathscr{R}=\mathscr{U} \mathscr{M}:=\{N \mid N$ has no non-zero homomorphic image in $\mathscr{M}\}$.

From Holcombe [6] and Groenewald [5] we need the following definitions.

For $i \in\{0,1,2\}$, a near-ring $N$ is called $i$-prime if $A B=0$ for $A$, $B \subseteq N$ implies $A=0$ or $B=0$ where, for

$i=0, A$ and $B$ are ideals of $N$ (this is just the usual definition of primeness for near-rings),

$i=1, A$ and $B$ are left ideals of $N$,

$i=2, A$ and $B$ are $N$-subgroups of $N$.

A near-ring $N$ is called 3-prime if $a N b=0 \quad(a, b \in N)$ implies a $a=0$ or $b=0$.

It is clear that $i$-prime implies $(i-1)$-prime for $i=1,2,3$ and, as is well known, for rings they all coincide. Our interest here will focus on 2-prime and 3-prime. It can easily be verified that if $N$ is a near-rings with a left identity, then $N$ is 2-prime if and only if it is 3-prime.

1.2 Definition. A class of near-rings $\mathscr{M}$ is called a special class if it satisfies the following conditions:

(S1) every $N \in \mathscr{M}$ is 2-prime and reliable;

(S2) $\mathscr{M}$ is hereditary;

(S3) $\mathscr{M}$ is closed under essential extensions.

1.3 Proposition. The following are equivalent for a class of near-rings $\mathscr{H}:$

(1) $\mathscr{M}$ is a special class;

(2) $\mathscr{M}$ is a hereditary class of 2-prime near-rings which is closed under essential extensions and satisfies condition

(F) $\quad$ if $J \triangleleft I \triangleleft N$ and $I / J \in \mathscr{M}$, then $J \triangleleft N$;

(3) $\mathscr{M}$ is a hereditary class of 2-prime reliable near-rings such that if $I \triangleleft N$ and $I \in \mathscr{M}$, then $N /(0: I)_{N} \in \mathscr{M}$;

(4) $\mathscr{M}$ is a hereditary class of 2-prime reliable near-rings such that if $I \triangleleft N$ and $I \in \mathscr{M}$ with $(0: I)_{N}=0$, then $N \in \mathscr{M}$. 
Proof. The equivalence of (1) and (2) can be found in [17]; the implications $(3) \rightarrow(4) \rightarrow(1)$ are straightforward. We show that $(1) \Rightarrow(3)$. Let $I \triangleleft N$ with $I \in \mathscr{M}$ and $(0: I)_{N}=0$. Then $I$ is an essential ideal in $N$ and $N \in \mathscr{M}$ follows from (1).

Any class of prime rings satisfies condition $(F)$ in the variety of rings: hence the special class defined above coincides with the usual definition of a special class in this variety. A near-ring $N$ is equiprime [4] if anx = any for all $n \in N$ where $0 \neq a \in N, x, y \in N$, implies $x=y$. Such near-rings are 3-prime as well as quasi semi-equiprime. Consequently any hereditary class of equiprime near-rings which is closed under essential extensions in a special class. Several such examples can be found in [17], and amongst these are the 3-primitive near-rings. Further examples are the class of 2-primitive near-rings and the class of simple near-rings with identity.

As can be expected, there is a close relationship between weakly special classes and special classes: the latter always implies the former since 1-prime and reliable implies qausi semi-equiprime. From [17, Theorem 2.10] we know that the subdirect closure of a weakly special class is again a weakly special class; hence we have

1.4 Proposition. Let $\mathscr{M}$ be a special class. Then the subdirect closure $\overline{\mathscr{M}}:=\{N \mid N$ is a subdirect sum of near-rings from $\mathscr{M}\}$ of $\mathscr{M}$ is a weakly special class.

1.5 Definition. A radical class $\mathscr{R}$ is called a special radical class if $\mathscr{R}$ is the upper radical determined by a special class.

As usual, for a radical class $\mathscr{R}$ and a near-ring $N, \mathscr{R}(N)$ denotes the radical of $N$ with respect to $\mathscr{R}$, that is, $\mathscr{R}(N)=\sum(I \triangleleft N \mid I \in \mathscr{R})$. Since any special radical class is an overnilpotent radical, we have from [17]

1.6 Theorem. Let $\mathscr{R}$ be a special radical class, say $\mathscr{R}=\mathscr{U} \mathscr{M}$ where $\mathscr{M}$ is a special class. Then $\mathscr{R}$ is ideal-hereditary, that is, $\mathscr{R}(I)=\mathscr{R}(N) \cap I$ for all $I \triangleleft N, \mathscr{S R}=\overline{\mathscr{M}}$ and $\mathscr{R}(N)=\bigcap\{I \triangleleft N \mid N / I \in \mathscr{M}\}$ for all near-rings $N$.

In the variety of rings there are non-special supernilpotent radical classes; the same holds for our generalizations, that is, there are non-special overnilpotent radicals in the variety of near-rings. This is not an immediate consequence of the ring case, since a class of rings which is closed under essential extensions in the variety of rings need not have this property in the bigger 
variety of all near-rings. However, an example of a non-special supernilpotent radical in the variety of rings given by Van Leeuwen and Jenkins [16] can be adapted for our case, as follows.

Let $S$ be a simple ring with identity which is not a field. In particular, $S$ is quasi semi-equiprime. Let $\mathscr{T}=\{A \mid A$ is a subdirect sum of copies of $S$ and $S$ is not and ideal of $A\}$. The class $\mathscr{T}$ is not empty; Van Leeuwen and Jenkins exhibited a ring $S$ as above and a ring $A$ in $\mathscr{T}$ which has only one non-zero prime homomorphic image $B$. This $B$ is a simple ring with identity, it is not a field and neither is $B$ in $\mathscr{T}$. Let $\mathscr{M}=\mathscr{T} \cup\{F \mid F$ is a field $\}$. Since the variety of rings is a subvariety of the variety of all near-rings, $\mathscr{M}$ consists of rings. We show that $\mathscr{K}$ is a weakly special class in the variety of all near-rings. Clearly all elements of $\mathscr{M}$ are quasi semi-equiprime and the hereditariness of $\mathscr{K}$ follows as in [16]. Finally we have to show that if $D$ is a near-ring and $C \triangleleft D$ with $C \in \mathscr{M}$ and $(0: C)_{D}=0$, then $D \in \mathscr{M}$. But the proof of this property for rings given by van Leeuwen and Jenkins [16] can readily be adapted for the present near-ring case; hence $\mathscr{M}$ is a weakly special class in the variety of near-rings and consequently $\mathscr{R}=\mathscr{U} \mathscr{M}$ is an overnilpotent radical. If $\mathscr{R}$ is a special radical, then $\mathscr{R}=\mathscr{U} \mathscr{K}$ for some special class $\mathscr{K}$. Since $A \in \mathscr{T} \subseteq \mathscr{M} \subseteq \overline{\mathscr{M}}=\mathscr{S} \mathscr{R}=\mathscr{S} \mathscr{U} \mathscr{K}, A$ has a nonzero homomorphic image in $\mathscr{K}$. Since $A \in \mathscr{T} \subseteq \mathscr{M} \subseteq \overline{\mathscr{M}}=\mathscr{S} \mathscr{R}=\mathscr{S} \mathscr{U} \mathscr{K}$, $A$ has a non-zero homomorphic image in $\mathscr{K}$ which must be a prime ring. But $A$ has only one such homomorphic image, namely $B$ and $B \notin \mathscr{K}$. Since $B$ is simple and not in $\mathscr{P} \mathscr{R}$, it follows that $B \in \mathscr{R} \cap \mathscr{K}=0$ which is not possible. Hence $\mathscr{R}$ is not a special radical.

As is well known, the variety of rings has a largest special class (namely the class of all prime rings) and consequently a smallest special radical. The status of this result for near-rings is not known.

\section{Near-ring groups and special radicals}

Analogous to the overnilpotent radical case [18], we have a characterization of special radicals via near-ring groups. For each near-ring $N$, let $\mathscr{K}_{N}$ be a (possibly empty) class of $N$-groups. Let $\mathscr{K}=\bigcup_{N} \mathscr{K}_{N}$.

2.1 Definition. $\mathscr{K}$ is a special class of near-rings groups if it satisfies the following conditions.

(SG1) $G \in \mathscr{K}_{N}, I \triangleleft N$ with $I G=0$ implies $G \in \mathscr{K}_{N / I}($ via $(n+I) g=n g)$.

(SG2) If $I \triangleleft N$ and $g \in \mathscr{K}_{N / I}$, then $G \in \mathscr{K}_{N}$ (via $n g=(n+I) g$ ).

(SG3) $G \in \mathscr{K}_{N}, I \triangleleft N$ and $I G \neq 0$ implies $G \in \mathscr{K}_{I}$. 
(SG4) $G \in \mathscr{K}_{N}$ implies $N G \neq 0$ and $N /(0: G)_{N}$ is a 2-prime reliable nearring.

(SG5) If $I \triangleleft N$ and $G \in \mathscr{K}_{I}$, then there exists an $N$-group $H \in \mathscr{K}_{N}$ with $(0: H)_{I} \subseteq(0: G)_{I}$.

Let $\mathscr{M}(\mathscr{K})=\left\{N \mid\right.$ there exists a faithful $N$-group $\left.G \in \mathscr{K}_{N}\right\}$.

If we replace "2-prime and reliable" in condition (SG4) above with "quasi semi-equiprime", then we obtain the definition of a weakly special class of near-ring groups used in [18] to characterize the overnilpotent radicals of near-rings. Since any quasi semi-equiprime near-ring is reliable, the proof of the next result follows mutatis mutandis as the corresponding one in [18] for weakly special classes and is therefore omitted.

2.2 THeOREM. Let $\mathscr{K}$ be a special class of near-ring groups. Then $\mathscr{M}(\mathscr{K})$ is a special class of near-rings and $\mathscr{R}=\mathscr{U} \mathscr{M}(\mathscr{K})$ is a special radical such that $\mathscr{R}(N)=\bigcap\left\{(0: G)_{N} \mid G \in \mathscr{K}_{N}\right\}$ for all near-rings $N$. Conversely, if $\mathscr{R}$ is a special radical class, say $\mathscr{R}=\mathscr{U} \mathscr{M}$ where $\mathscr{M}$ is a special class, then there is a special class of near-ring groups $\mathscr{K}$ such that $\mathscr{M}=\mathscr{M}(\mathscr{K})$.

\section{Radicals and matrix near-rings}

In this section we investigate the relationship between the radical of a nearring $N$ and the radical of the matrix near-ring $\mathbf{M}_{n}(N)$ over $N$. First we recall some relevant definitions and properties concerning matrix near-rings from Meldrum and Van der Walt [9], Van der Walt [15] and Meyer [10].

In the sequel, $R$ will always be a near-ring with identity 1 . Let $R^{n}$ be the direct sum of $n$ copies $(n \geq 2)$ of the underlying group of $R$. The elements of $R^{n}$ will be written in pointed brackets as $\left\langle r_{1}, r_{2}, \ldots, r_{n}\right\rangle$ or sometimes just as $r$. The $n \times n$ matrix near-ring $\mathbf{M}_{n}(R)$ over $R$ is the subnearring of $\mathbf{M}_{0}\left(R^{n}\right)=\left\{\right.$ all functions $f: R^{n} \rightarrow R^{n}$ for which $f\langle 0,0, \ldots, 0\rangle=$ $\langle 0,0, \ldots, 0\rangle\}$ generated by the set of functions $\left\{f_{i j}^{a} \mid 1 \leq i, j \leq n ; a \in R\right\}$ where $f_{i j}^{a}\left\langle r_{1}, r_{2}, \ldots, r_{n}\right\rangle=\left\langle t_{1}, t_{2}, \ldots, t_{n}\right\rangle$ with $t_{k}=0$ for $k \neq i$ and $t_{i}=a r_{j}$. The near-ring $\mathbf{M}_{n}(R)$ is a $o$-symmetric near-ring with identity $I=f_{11}^{1}+f_{22^{n}}^{1}+\cdots+f_{n n}^{1}$. If $R$ is a ring, then $\mathbf{M}_{n}(R)$ is the ring of all $n \times n$ matrices over $R$. Elements of $\mathbf{M}_{n}(R)$ are called matrices.

For $A \triangleleft R$, both $A^{*}=\left\{U \in \mathbf{M}_{n}(R) \mid U r \in A^{n}\right.$ for all $\left.r \in R^{n}\right\}$ and $A^{+}=$ $\operatorname{id}\left\{f_{i j}^{a} \mid a \in A, 1 \leq i, j \leq n\right\}$ are ideals in $\mathbf{M}_{n}(R)$. Here $A^{n}$ is $A \times A \times \cdots \times A$ ( $n$ factors) and id $\{\ldots\}$ is the ideal in $\mathbf{M}_{n}(R)$ generated by $\{\ldots\}$. Note that $A^{+}$is always contained in $A^{*}$ (for rings they coincide, but for near-rings the inclusion can be strict) and both the maps $A \longmapsto A^{*}$ and $A \longmapsto A^{+}$are 
injections. If $\mathscr{A} \triangleleft \mathbf{M}_{n}(R)$, then $A_{*}=\left\{a \in R \mid a=t_{i}\right.$ for some $i$ where $U r=t$ for some $U \in \mathscr{A}$ and $\left.r \in R^{n}\right\}$. For ideals $\mathscr{A} \triangleleft \mathbf{M}_{n}(R)$ and $A$, $B \triangleleft R$, the following connections hold:

(i) $\left(\mathscr{A}_{*}\right)^{+} \subseteq \mathscr{A} \subseteq\left(\mathscr{A}_{*}\right)^{*}$;

(ii) $\left(A^{+}\right)_{*}=A=\left(A^{*}\right)_{*}$;

(iii) $\left(\left(\mathscr{A}_{*}\right)^{+}\right)_{*}=\left(\left(A_{*}\right)^{*}\right)_{*}$;

(iv) $A \subseteq B$ implies $A^{+} \subseteq B^{+}$and $A^{*} \subseteq B^{*}$;

(v) $A^{+} \subseteq B^{*}$ implies $A \subseteq B$; hence $A^{*} \subseteq B^{*}$ implies $A \subseteq B$;

(vi) $(A \cap B)^{+} \subseteq A^{+} \cap B^{+}$;

(vii) $\left(A \cap B^{*}\right)=A^{*} \cap B^{*}$ (this can be extended to arbitrary intersections).

A matrix $U$ is a $k$ th column matrix if it is of the form

$$
U=f_{1 k}^{a}+f_{2 k}^{b}+\cdots+f_{n k}^{c}
$$

for some $a, b, \ldots, c \in R$. We denote by $C_{k}$ the set of all $k$ th column matrices of $\mathbf{M}_{n}(R)$. Every $C_{k}$ is an $\mathbf{M}_{n}(R)$-subgroup of $\mathbf{M}_{n}(R)$.

For $\mathscr{T} \triangleleft \mathbf{M}_{n}(R), U \in\left(\mathscr{T}_{*}\right)^{*}$ if and only if $f_{i j}^{1} U\left(f_{1 k}^{a}+f_{2 k}^{b}+\cdots+f_{n k}^{c}\right) \in \mathscr{T}$ for all $1 \leq i, j, k \leq n$ and $a, b, \ldots, c \in R$. In general $\mathscr{T} \subseteq\left(\mathscr{T}_{*}\right)^{*}$ (for rings they coincide).

3.1 Proposition. Let $\mathscr{T} \triangleleft \mathbf{M}_{n}(R)$ and let $U \in \mathbf{M}_{n}(R)$. Then the following are equivalent:

(1) $\mathscr{T}=\left(\mathscr{T}_{*}^{*}\right.$

(2) $U C_{1} \subseteq \mathscr{T}$ implies $U \in \mathscr{T}$;

(3) $U \mathbf{M}_{n}(R) f_{11}^{1} \subseteq \mathscr{T}$ implies $U \in \mathscr{T}$;

(4) $U \mathbf{M}_{n}(R) f_{k k}^{1} \subseteq \mathscr{T}$ for some $k \quad(1 \leq k \leq n)$ implies $U \in \mathscr{T}$.

Proof. (1) $\Rightarrow$ (2). If $U C_{1} \subseteq \mathscr{T}$ but $U \notin \mathscr{T}=\left(\mathscr{T}_{*}\right)^{*}$, then $f_{i j}^{1} U\left(f_{1 k}^{a}+f_{2 k}^{b}+\cdots+f_{n k}^{c}\right) \notin \mathscr{T}$ for some $i, j, k \in\{1,2, \ldots, n\}$ and $a$, $b, \ldots, c \in R$. This contradicts $U C_{1} \subseteq \mathscr{T}$ since $f_{i j}^{1} U\left(f_{1 k}^{a}+f_{2 k}^{b}+\cdots+f_{n k}^{c}\right)=$ $f_{i j}^{1} U\left(f_{11}^{a}+f_{21}^{b}+\cdots+f_{n 1}^{c}\right) f_{1 k}^{1}$.

(2) $\Rightarrow(3)$. This is obvious since $\mathbf{M}_{n}(R) f_{11}^{1}=C_{1}$.

(3) $\Rightarrow(4)$. This is obvious since $f_{11}^{1}=f_{1 k}^{1} f_{k k}^{1} f_{k 1}^{1}$ for any $k$.

(4) $\Rightarrow(1)$. Assume the validity of the implication for some $k$ and let $U \in\left(\mathscr{T}_{*}^{*}\right.$ but with $U \notin \mathscr{T}$. Then $U V f_{k k}^{1} \notin \mathscr{T}$ for some $V \in \mathbf{M}_{n}(R)$. Now $V f_{k k}^{1}=f_{1 k}^{a}+f_{2 k}^{b}+\cdots+f_{n k}^{c}$ for some $a, b, \ldots, c \in R$ (cf. [9, Lemma 3.7]). Furthermore, $f_{i i}^{1} U V f_{k k}^{1}=f_{i i}^{1} U\left(f_{1 k}^{a}+f_{2 k}^{b}+\cdots+f_{n k}^{c}\right) \in \mathscr{T}$ since $U \in$ $\left(\mathscr{T}_{*}\right)^{*}$. Consequently $U \in \mathscr{T}$. 
It is clear that if $\mathscr{T} \triangleleft \mathbf{M}_{n}(R)$ and $f_{k k}^{1} \in \mathscr{T}$ for some $k$, then $\mathscr{T}=\mathbf{M}_{n}(R)$. Moreover, since any 2-prime near-ring with an identity is 3-prime, we have

3.2 Corollary. If $\mathscr{T} \triangleleft \mathbf{M}_{n}(R)$ and $\mathbf{M}_{n}(R) / \mathscr{T}$ is 2-prime, then $\mathscr{T}=$ $\left(\mathscr{T}_{*}\right)^{*}$

Of course, the converse is not true since the equality $\mathscr{T}=\left(\mathscr{T}_{*}\right)^{*}$ holds for any ring $R$. The ideals $\mathscr{T} \triangleleft \mathbf{M}_{n}(R)$ for which $\mathscr{T}=\left(\mathscr{T}_{*}\right)^{*}$ are called full ideals of $\mathbf{M}_{n}(R)$.

In the sequel, $\mathscr{M}$ will be a class of near-rings, always assumed to be abstract (that is, contains the one element near-ring and all isomorphic copies of near-rings in $\mathscr{M}$ ). With $\mathscr{M}$ is associated the corresponding Hoehnke radical $\mathscr{L}$ defined by $\mathscr{L}(N=\bigcap\{A \mid A \triangleleft N, N / A \in \mathscr{M}\}$. A Hoehnke radical need not be a Kurosh-Amitsur radical. For a detailed discussion on the relationship between these two types of radicals, [11] can be consulted. However, we recall that if $\mathscr{L}$ is the Hoehnke radical associated with $\mathscr{M}$, then $\mathscr{L}(N)=0$ if and only if $N \in \overline{\mathscr{H}}$ where $\overline{\mathscr{M}}$ is the subdirect closure of $\mathscr{M}$. For ease of reference, if $A \triangleleft N$, and $N / A \in \mathscr{M}, A$ is called an $\mathscr{M}$-ideal of $N$ and $(N) \mathscr{K}$ denotes the intersection of all the $\mathscr{K}$-ideals of $N$, that is $(N) \mathscr{M}=\bigcap\{A \mid A \triangleleft N, N / A \in \mathscr{M}\}$. For any subclass $\mathscr{K} \subseteq \mathscr{M}$ with $\overline{\mathscr{K}}=\overline{\mathscr{H}}$, we have $(N) \mathscr{K}=(N) \overline{\mathscr{K}}=(N) \mathscr{K}$. For any Kurosh-Amitsur radical class $\mathscr{R}$, the Hoehnke radical $\mathscr{L}$ determined by

$$
\mathscr{L}(N)=\bigcap(A \mid A \triangleleft N, N / A \in \mathscr{S} \mathscr{R}\}
$$

coincides with $\mathscr{R}(N)$ and is thus a Kurosh-Amitsur radical.

3.3 Proposition. Let $\mathscr{M}$ be a class of near-rings, $\mathscr{L}$ the associated Hoehnke radical.

If for each near-ring with identity $N, N \in \mathscr{M}$ implies $\mathbf{M}_{n}(R) \in \mathscr{M}$, then $\mathscr{L}\left(\mathbf{M}_{n}(R)\right) \subseteq(\mathscr{L}(R))^{*}$.

Proof. Let $A \triangleleft R$ with $R / A \in \mathscr{M}$. From [14, Lemma 4.2] we have $\mathbf{M}_{n}(R) / A^{*} \cong \mathbf{M}_{n}(R / A) \in \mathscr{M}$. From this and [12,1.44], we get

$$
\begin{aligned}
\mathscr{L}\left(\mathbf{M}_{n}(R)\right) & =\bigcap\left\{\mathscr{T} \triangleleft \mathbf{M}_{n}(R) \mid \mathbf{M}_{n}(R) / \mathscr{T} \in \mathscr{M}\right\} \\
& \subseteq \bigcap\left\{A^{*} \mid A \triangleleft R, R / A \in \mathscr{M}\right\} \\
& =(\bigcap\{A \mid A \triangleleft R, R / A \in \mathscr{M}\})^{*} \\
& =(\mathscr{L}(R))^{*} .
\end{aligned}
$$

If $\mathscr{M}$ is closed under subdirect sums, that is, $\mathscr{M}=\overline{\mathscr{M}}$, then the converse is clearly valid. This, in addition with $(N) \mathscr{K}=(N) \overline{\mathscr{K}}$ for arbitrary $\mathscr{M}$ yields 
3.4 Corollary. Let $\mathscr{M}$ be a class of near-rings, $\mathscr{L}$ the associated Hoehnke radical. Then $\mathscr{L}\left(\mathbf{M}_{n}(R)\right) \subseteq(\mathscr{L}(R))^{*}$ for all near-rings $R$ with identity if and only if $\mathscr{M}$ satisfies the following condition: for each near-ring with identity $N, N \in \overline{\mathscr{M}}$ implies $\mathbf{M}_{n}(R) \in \overline{\mathscr{M}}$.

The class $\mathscr{K}$ is said to have the matrix extension property if it satisfies the condition: whenever $N$ is a near-ring with identity, then $N \in \mathscr{M}$ if and only if $\mathbf{M}_{n}(N) \in \mathscr{M}$. This however, contrary to the ring case, is not enough to ensure the equality in Proposition 3.3.

3.5 THEOREM. Let $\mathscr{M}$ be a class of near-rings, $\mathscr{L}$ the associated Hoehnke radical. If every $\mathscr{M}$-deal of $\mathbf{M}_{n}(R)$ is full and $\mathscr{M}$ has the matrix extension property, then $\mathscr{L}\left(\mathbf{M}_{n}(R)\right)=(\mathscr{L}(R))^{*}$.

Proof. Let $\mathscr{T}$ be an $\mathscr{M}$-ideal of $\mathbf{M}_{n}(R)$. Then $\mathscr{T}_{*} \triangleleft R$ and $\mathbf{M}_{n}\left(R / \mathscr{T}_{*}\right) \cong$ $\mathbf{M}_{n}(R) /\left(\mathscr{T}_{*}\right)^{*}=\mathbf{M}_{n}(R) / \mathscr{T} \in \mathscr{M}$; hence $\mathscr{T}_{*}$ is an $\mathscr{M}$-ideal of $R$. Thus

$$
\begin{aligned}
(\mathscr{L}(R))^{*} & =(\bigcap\{A \mid A \triangleleft R, R / A \in \mathscr{M}\})^{*} \\
& =\bigcap\left\{A^{*} \mid A \triangleleft R, R / A \in \mathscr{K}\right\} \\
& \subseteq \bigcap\left\{\mathscr{T} \mid \mathscr{T} \triangleleft \mathbf{M}_{n}(R), \mathbf{M}_{n}(R) / \mathscr{T} \in \mathscr{M}\right\} \\
& =\mathscr{L}\left(\mathbf{M}_{n}(R)\right)
\end{aligned}
$$

and the equality follows from Proposition 3.3.

If $\mathscr{M}$ is closed under subdirect sums, then $\mathscr{L}\left(\mathbf{M}_{n}(R)\right)=(\mathscr{L}(R))^{*}$ for all near-rings $R$ with identity, implies that $\mathscr{M}=\overline{\mathscr{M}}$ has the matrix extension property. Hence we have

3.6 Corollary. Let $\mathscr{M}$ be such that every $\mathscr{H}$-ideal of $\mathbf{M}_{n}(R)$ is full for all near-rings $R$ with identity. Then $\mathscr{L}\left(\mathbf{M}_{n}(R)\right)=(\mathscr{L}(R))^{*}$ for all $R$ (with identity) if and only if $\overline{\mathscr{M}}$ has the matrix extension property.

PRoof. It only has to be verified that if every $\mathscr{M}$-ideal of $\mathbf{M}_{n}(R)$ is full, then every $\overline{\mathscr{M}}_{\text {-ideal of }} \mathbf{M}_{n}(R)$ is also full. But this follows from

3.7 Lemma. Any intersection of full ideals of $\mathbf{M}_{n}(R)$ is a full ideal of $\mathbf{M}_{n}(R)$.

Proof. The following will suffice to verify the proof: for any set of ideals $\left\{A_{a}\right\}$ of $R, \bigcap_{a}\left(A_{a}\right)^{*}=\left(\bigcap_{a} A_{\alpha}\right)^{*}$ and if $\mathscr{T} \triangleleft \mathbf{M}_{n}(R)$, then $a \in \mathscr{T}_{*}$ if and only if $f_{11}^{a} \in \mathscr{T}$ (cf. [9] and [14]). 
Concerning special classes $\mathscr{M}$, we have

3.8 THEOREM. Let $\mathscr{M}$ be a special class of near-rings which satisfies the matrix extension property. Let $\mathscr{R}$ be the corresponding special radial. For any near-ring $N$ with identity and any $A \triangleleft N$,

$$
(\mathscr{R}(A))^{+} \subseteq \mathscr{R}\left(A^{+}\right) \subseteq \mathscr{R}\left(A^{*}\right)=(\mathscr{R}(A))^{*} .
$$

In particular $($ for $A=N), \mathscr{R}\left(\mathbf{M}_{n}(N)\right)=(\mathscr{R}(N))^{*}$.

Proof. From Theorem 1.6, $\mathscr{R}(N)=(N) \mathscr{M}$ and $\mathscr{R}$ is ideal-hereditary. Corollary 3.2 and Theorem 3.5 give $\mathscr{R}\left(\mathbf{M}_{n}(N)\right)=(\mathscr{R}(N))^{*}$. For any $A \triangleleft N$ and $\mathscr{T} \triangleleft \mathbf{M}_{n}(N)$, we have $\mathscr{R}(\mathscr{T})=\mathscr{T} \cap \mathscr{R}\left(\mathbf{M}_{n}(N)\right)$ and $\mathscr{R}(A)=A \cap \mathscr{R}(N)$; hence

$$
\begin{aligned}
(\mathscr{R}(A))^{+} & =(A \cap \mathscr{R}(N))^{+} \subseteq A^{+} \cap(\mathscr{R}(N))^{+} \subseteq A^{+} \cap(\mathscr{R}(N))^{*} \\
& =A^{+} \cap \mathscr{R}\left(\mathbf{M}_{n}(N)\right)=\mathscr{R}\left(A^{+}\right) \subseteq \mathscr{R}\left(A^{*}\right)=A^{*} \cap \mathscr{R}\left(\mathbf{M}_{n}(N)\right) \\
& =A^{*} \cap(\mathscr{R}(N))^{*}=(A \cap \mathscr{R}(N))^{*}=(\mathscr{R}(A))^{*} .
\end{aligned}
$$

3.9 Corollary. Let $\mathscr{M}$ and $\mathscr{R}$ be as above. Let $A \triangleleft N, \mathscr{T} \triangleleft \mathbf{M}_{n}(N)$. Then

(1) $A \in \mathscr{R}$ if and only if $A^{*} \in \mathscr{R}$, which holds if and only if $A^{+} \in \mathscr{R}$,

(2) $A \in \mathscr{S R}$ if and only if $A^{*} \in \mathscr{S R}$, which holds if and only if $A^{+} \in$ $\mathscr{P S}$

(3) $\mathscr{T} \in \mathscr{R}$ if and only if $\mathscr{T}_{*} \in \mathscr{R}$,

(4) $\mathscr{T} \in \mathscr{S} R$ if and only if $\mathscr{T}_{*} \in \mathscr{S} \mathscr{R}$.

Proof. Since $\mathscr{R}$ is ideal-hereditary, both $\mathscr{R}$ and $\mathscr{S} \mathscr{R}$ are hereditary and we will repeatedly use the fact that if $C, D \triangleleft K$ for a near-ring $K$ with $C \subseteq D$, then $D \in \mathscr{R}$ implies $C \in \mathscr{R}$ and $D \in \mathscr{S} \mathscr{R}$ implies $C \in \mathscr{S} \mathscr{R}$.

(1) Let $A \in \mathscr{R}$. Then $A \subseteq \mathscr{R}(N)$ and so $A^{+} \subseteq(\mathscr{R}(N))^{+} \subseteq(\mathscr{R}(N))^{*}$ and $A^{*} \subseteq(\mathscr{R}(N))^{*}$. Since $(\mathscr{R}(N))^{*}=\mathscr{R}\left(\mathbf{M}_{n}(R)\right) \in \mathscr{R}$, we have $A^{+}$and $A^{*}$ both in $\mathscr{R}$. If $A^{*} \in \mathscr{R}$, then $A^{+} \in \mathscr{R}$ since $A^{+} \subseteq A^{*}$. If $A^{+} \in \mathscr{R}$, then $A^{+} \subseteq \mathscr{R}\left(\mathbf{M}_{n}(R)\right)=(\mathscr{R}(N))^{*}$; hence $A \subseteq \mathscr{R}(N) \in$ $\mathscr{R}$ which gives $A \in \mathscr{R}$.

(2) If $A \in \mathscr{S} \mathscr{R}$, then $\mathscr{R}\left(A^{+}\right) \subseteq \mathscr{R}\left(A^{*}\right)=(\mathscr{R}(A))^{*}=0$, that is, $A^{+}$ and $A^{*}$ are both in $\mathscr{S} \mathscr{R}$. For $A^{*} \in \mathscr{S} \mathscr{R},(\mathscr{R}(A))^{*}=\mathscr{R}\left(A^{*}\right)=0$, that is, $\mathscr{R}(A)=0$ and $A \in \mathscr{S} \mathscr{R}$. If $A^{+} \in \mathscr{S} \mathscr{R}$, then $(\mathscr{R}(A))^{+} \subseteq$ $\mathscr{R}\left(A^{+}\right)=0$, that is $\mathscr{R}(A)=0$ and $A \in \mathscr{P} \mathscr{R}$.

(3) $\mathscr{T} \in \mathscr{R}$ implies $\mathscr{T} \subseteq \mathscr{R}\left(\mathbf{M}_{n}(R)\right)=(\mathscr{R}(N))^{*}$. Hence $\left.\mathscr{T} \subseteq(\mathscr{R}(N))^{*}\right)_{*}$ $=\mathscr{R}(N)$ and $\mathscr{T}_{*} \in \mathscr{R}$. If $\mathscr{T}_{*} \in \mathscr{R}$, then $\mathscr{T}_{*} \subseteq \mathscr{R}(N)$ and thus $\mathscr{T} \subseteq\left(\mathscr{T}_{*}\right)^{*} \subseteq(\mathscr{R}(N))^{*} \in \mathscr{R} ;$ hence $\mathscr{T} \in \mathscr{R}$. 
(4) $\mathscr{T} \in \mathscr{S} \mathscr{R}$ and $\left(\mathscr{F}_{*}\right)^{+} \subseteq \mathscr{T}$ implies $\left(\mathscr{T}_{*}\right)^{+} \in \mathscr{S} \mathscr{R}$. Hence $\left(\mathscr{R}\left(\mathscr{T}_{*}\right)\right)^{+}$ $\subseteq \mathscr{R}\left(\left(\mathscr{T}_{*}\right)^{+}\right)=0$; that is, $\mathscr{T}_{*} \in \mathscr{S} \mathscr{R}$. If $\mathscr{T}_{*} \in \mathscr{S} \mathscr{R}$, then $\mathscr{R}(\mathscr{T})$ $\subseteq \mathscr{R}\left(\left(\mathscr{T}_{*}\right)^{*}\right)=0$. Thus $\mathscr{T} \in \mathscr{P} \mathscr{R}$.

In conclusion we recall the known results on the relationship between the radicals of near-rings and the corresponding matrix near-rings. If $\mathscr{H}_{\nu}$ is the class of $\nu$-primitive near-rings $(\nu \in\{0,1,2,3\})$, we only consider the cases $\nu=0$ and $\nu=2$ since 1-primitivity, 2-primitivity and 3-primitivity coincide on near-rings with identity. The class of 2-primitive near-rings has the matrix extension property (Van der Walt [14]) and if $R$ is 0-primitive, then so is $\mathbf{M}_{n}(R)$. The converse is not true and $J_{0}\left(\mathbf{M}_{n}(R)\right)$ can be strictly contained in $\left(J_{0}(R)\right)^{*}$ (Meldrum and Meyer [8]).

Both the classes of equiprime near-rings and strongly equiprime near-rings respectively are special (in our sense) and have the matrix extension property (Booth and Groenewald [2]). The classes of prime (that is, 0-prime), respectively semi-prime, near-rings have the matrix extension property (Meyer [10]). Thus, if $\mathscr{P}$ is the corresponding radical, then $\mathscr{P}\left(\mathbf{M}_{n}(R)\right) \subseteq(\mathscr{P}(R))^{*}$. Equality need not hold in this case: in [8] Meldrum and Meyer presented an example of a finite near-ring $R$ for which $J_{0}\left(\mathbf{M}_{n}(R)\right) \subset\left(J_{0}(R)\right)^{*}$. Since $R$ is finite, both $R$ and $\mathbf{M}_{n}(R)$ have the DCCN and from Pilz [12, 5.61], we have $\mathscr{P}\left(\mathbf{M}_{n}(R)\right)=J_{0}\left(\mathbf{M}_{n}(R)\right) \subset\left(J_{0}(R)\right)^{+}=(\mathscr{P}(R))^{+}$. In particular, does this show that a prime ideal of $\mathbf{M}_{n}(R)$ need not be full. The class $\mathscr{M}$ of 3-prime near-rings has the matrix extension property and every $\mathscr{M}$-ideal of $\mathbf{M}_{n}(R)$ is full (Groenewald [5]). Hence in this case $\mathscr{L}\left(\mathbf{M}_{n}(R)\right)=(\mathscr{L}(R))^{*}$. It is not known whether this class is a special class; in fact it is not even known whether the corresponding radical $\mathscr{L}$ is a Kurosh-Amitsur radical.

Finally, the class of simple near-rings with identity is a special class and has the matrix extension property (Meldrum and Van der Walt [9]).

\section{References}

[1] T. Anderson, K. Kaarli and R. Wiegandt, 'Radicals and subdirect decomposition', Comm. Algebra 13 (1985), 479-494.

[2] G. L. Booth and N. J. Groenewald, 'On primeness in matrix near-rings', Arch. Math., 56 (1991), 539-546.

[3] G. L. Booth and N. J. Groenewald, 'Special radicals near-rings', Math. Japon. to appear.

[4] G. L. Booth, N. J.Groenewald and S. Veldsman, 'A Kurosh-Amitsur prime radical for near-rings', Comm. Algebra 18 (1990), 3111-3122.

[5] N. J. Groenewald, 'Different prime ideals in near-rings', Comm. Algebra 19 (1991), 2667-2675.

[6] W. M. L. Holcombe, Primitive near-rings, Ph. D. Thesis, University of Leeds, 1970. 
[7] K. Kaarli, 'Classification of irreducible $R$-groups over a semiprimary near-ring', Tartu Rikk. Ul. Toimetised 556 (1981), 47-63 (in Russian).

[8] J. D. P. Meldrum and J. H. Meyer, 'Modules over matrix near-rings and the $J_{0}$-radical', manuscript.

[9] J. D. P. Meldrum and A. P. J. van der Walt, 'Matrix near-rings', Arch. Math. 47 (1986), 312-319.

[10] J. H. Meyer, Matrix near-rings, Ph. D. Thesis, University of Stellenbosch, 1986.

[11] R. Mlitz, and S. Veldsman, 'Radicals and subdirect decompositions in $\Omega$-groups', J. Austral. Math. Soc. 48 (1990), 171-198.

[12] G. Pilz, Near-rings, (North-Holland, Amsterdam, Revised Edition, 1983).

[13] F. A. Szasz, Radicals of Rings, (Akademiai Kiado, Budapest, 1981).

[14] A. P. J. van der Walt, 'Primitivity in matrix near-rings', Quaest. Math. 9 (1986), 459469.

[15] A. P. J. van der Walt, 'On two-sided ideals in matrix near-rings', in: Near-rings and Near-fields (Proc Conf. Tubingen 1985), G. Betsch (editor), Elsevier Science Publishers B. V. (North-Holland), 1987, 267-271.

[16] L. C. A. Van Leeuwen and T. O. Jenkins, 'A supernilpotent non-special radical class, Bull. Austral. Math. Soc. 9 (1973), 343-348.

[17] S. Veldsman, 'An overnilpotent radical theory for near-rings', J. Algebra 144 (1991), 248-265.

[18] S. Veldsman, 'On a characterization of overnilpotent radical classes of near-rings by $N$ groups', South African J. Science 87 (1991), 215-216.

University of Port Elizabeth

PO Box 1600

Port Elizabeth (6000)

South Africa 